\title{
Bio-Inspired Coating Strategies for the Immobilization of Polymyxins to Generate Contact-Killing Surfaces
}

\author{
Diana Alves, ${ }^{*}$ Maria Olívia Pereira
}

Microbial colonization of indwelling devices remains a major concern in modern healthcare. Developing approaches to prevent biomaterial-associated infections (BAI) is, therefore, in great demand. This study aimed to immobilize two antimicrobial peptides (polymyxins $B$ and $\mathrm{E}$ ) onto polydimethylsiloxane (PDMS) using two polydopamine (pDA)-based approaches: the conventional two-step method involving the deposition of a pDA layer to which biomolecules are immobilized, and a one-step method where peptides are dissolved together with dopamine before its polymerization. Surface characterization confirms the immobilization of polymyxins onto PDMS at a non-toxic concentration. Immobilization of polymyxins using a one-step pDA-based approach is able to prevent Pseudomonas aeruginosa adhesion and kill a significant fraction of the adherent ones. Living cells adhered to these modified surfaces exhibit the same susceptibility pattern as cells adhered to unmodified surfaces, highlighting no resistance development. Results suggest that polymyxins immobilization holds a great potential as an additional antimicrobial functionality in the design of biomaterials.

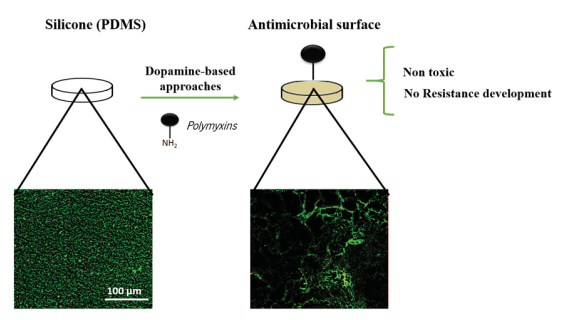

\section{Introduction}

Millions of lives are saved, every day in modern healthcare, thanks to the use of biomaterial implants and medical devices. Despite their crucial role in medicine progress, there are some drawbacks associated to their increased use as they all are prone to bacterial colonization..$^{[1,2]}$ Bacterial adhesion to an indwelling device, followed by biofilm formation, is commonly associated to persistent infections and subsequently to tissue destruction, systemic dissemination of the pathogens and dysfunction of the device,

Dr. D. Alves, Prof. M. O. Pereira

CEB-Centre of Biological Engineering

LIBRO-Laboratório de Investigação em Biofilmes

Rosário Oliveira

University of Minho

Campus de Gualtar

4710-057 Braga, Portugal

E-mail: dianalves@ceb.uminho.pt resulting in serious illness and death. ${ }^{[3]}$ Biomaterial-associated infections are extremely hard to treat because cells within a biofilm encase themselves in a self-produced polymeric matrix which confers them protection against antimicrobial treatment and host immune system. ${ }^{[4,5]}$ Among the microorganisms most frequently isolated from BAI, the Gram-negative Pseudomonas aeruginosa, stands out for its ability to form strong biofilms, ${ }^{[6,7]}$ intrinsic resistance to antibiotics, ${ }^{[8]}$ and remarkable ability to develop resistance during antimicrobial treatment. ${ }^{[9]}$

The development of materials that can resist or prevent bacterial adhesion constitutes the most promising approach to deal with BAI problem and modern biomaterial science has provided several modification and activation strategies to impart biomaterials with antibacterial properties. ${ }^{[10]}$ Different compounds such as antibiotics, ${ }^{[11]}$ quaternary ammonium compounds, ${ }^{[12]}$ and metal nanoparticles consisting of silver or metal oxides ${ }^{[13,14]}$ have been exploited to impart surfaces with antimicrobial features. However, most of these strategies present some limitations such as incomplete efficacy, toxicity, and the 
development of bacterial resistance. ${ }^{[15,16]}$ In the search for new compounds, antimicrobial peptides (AMP) have been recognized as promising candidates for the new generation of antimicrobial surfaces. ${ }^{[17,18]}$ AMP are a key component of the innate immune systems of most living organisms to protect them against invading microorganisms which mode of action involves electrostatic interaction with bacterial membranes with subsequent disruption of membrane's structural stability. ${ }^{[19]}$ Due to their attraction to negatively charged molecules on the bacterial membrane, acquisition of resistance to AMP in susceptible strains is slower and less common compared with that developed against other antimicrobials. ${ }^{[19,20]}$ Other advantages of AMP include their activity against a wide spectrum of microorganisms, efficacy at very low concentrations, and ability to enhance the antimicrobial action of classical antibiotics. ${ }^{[21]}$

Polymyxins are a group of cationic antimicrobial lipopeptides that has been used as the last resort to fight infections caused by multi-drug resistant $P$. aeruginosa strains. ${ }^{[22]}$ Only polymyxins B (PB) and E (PE) (also called colistin) have been used in clinical practice. ${ }^{[23]}$ Structurally, they consist of a seven-member cyclic ring of aminoacids with a tripeptide side chain bounded to a fatty acid chain. The two polymyxins have the same heptapeptide ring, with the exception of a single aminoacid, which is phenylalanine in PB and leucine in PE. ${ }^{[24]} \mathrm{Sev}$ eral studies have demonstrated the in vitro bactericidal activity of polymyxins alone and combined with other antimicrobials. ${ }^{[25,26]}$ Although effective, some concerns have been raised about the development of bacterial resistance and toxicity toward these AMP. ${ }^{[22]}$ Their covalent immobilization onto a biomaterial surface may overcome these drawbacks as it avoids patient exposure to sub-inhibitory concentrations. ${ }^{[27]}$

The aim of the current study was, thus, to immobilize two AMP (polymyxins B and E) onto PDMS, commonly referred as silicone rubber, which has been widely used for implantable biomedical devices such as catheters or voice prostheses, ${ }^{[28,29]}$ using dopamine chemistry. Two pDA-based approaches were compared: the conventional two-step method involving first the deposition of a pDA layer to which biomolecules are afterward immobilized, and a one-step method where compounds are incorporated throughout the full thickness of the pDA film as they are dissolved together with dopamine before its polymerization. ${ }^{[30]}$

\section{Experimental Section}

\subsection{Bacterial Strain and Growth Conditions}

A reference strain of $P$. aeruginosa (ATCC 39324) was used throughout this study. The strain was first streaked on a tryptic soy agar (TSA, Merck) plate from a frozen stock solution and grown for $24 \mathrm{~h}$ at $37^{\circ} \mathrm{C}$. For each experiment, a few colonies were collected from the TSA plates and grown overnight in batches of tryptic soy broth (TSB, Merck) at $37{ }^{\circ} \mathrm{C}$ under agitation (120 rpm). Subsequently, cells were harvested by centrifugation $(9000 \mathrm{~g}$, $5 \mathrm{~min}$, at room temperature) and washed three times in sterile saline solution $(0.9 \% \mathrm{NaCl}$ prepared in ultrapure water). The concentration of cellular suspensions was adjusted by optical density at $640 \mathrm{~nm}$ and calibrated in terms of Colony Forming Units (CFU) using spread plate method.

\subsection{Antimicrobial Peptides}

Two AMP were used throughout this work: polymyxin B (Biochrom) and polymyxin E (Colistin sulphate, Sigma).

\subsection{Antimicrobial Susceptibility of AMP}

The minimal inhibitory (MIC) and bactericidal (MBC) concentrations of peptides were determined by the microdilution method according to clinical and laboratory standards institute (formerly NCCLS) ${ }^{\left[{ }^{[1]}\right.}$ Briefly, the wells of a sterile 96 -well round-bottom microtiter plates (polystyrene, Orange, USA) were filled with $100 \mu \mathrm{L}$ of Mueller Hinton Broth (MHB, Merck) with increasing concentrations of peptide to which were added $100 \mu \mathrm{L}$ of each bacterium inoculum (adjusted to a final concentration of $5.0 \times 10^{5} \mathrm{CFU} \mathrm{mL}^{-1}$ ). The plates were afterward incubated at $37^{\circ} \mathrm{C}$ for $24 \mathrm{~h}$ in an orbital shaker at $120 \mathrm{rpm}$ (OS-20). In this assay, two controls were used, one without bacteria as a negative control and one without peptide as a positive control. Moreover, culture media with increasing concentrations of peptides without bacteria were also tested in order to avoid misleading results. The MIC of the planktonic fraction was obtained by measuring the absorbance at $640 \mathrm{~nm}$ $\left(A_{640 \mathrm{~nm}}\right)$ in an automated microtiter plate reader (Sunrise, Tecan), where clear wells $\left(A_{640 \mathrm{~nm}}=\right.$ negative control) were evidence of bacterial growth inhibition. MBC determination was performed by adding a droplet of $10 \mu \mathrm{L}$ from each well with no visible growth on a TSA plate. The lowest concentration that yielded no colony growth after $24 \mathrm{~h}$ at $37^{\circ} \mathrm{C}$ was identified as the MBC.

\subsection{PDMS Preparation}

PDMS was prepared by mixing and curing of two-component kit Sylgard 184 (Dow Corning, USA) at room temperature. Briefly, base and curing agents in the kit were mixed thoroughly in 10:1 (w/w), cast in a petri dish and kept at room temperature for $48 \mathrm{~h}$. After curing, PDMS formed in petri dish was cut into circle pieces of $0.9 \mathrm{~cm}$ diameter at a thickness of about $0.3 \mathrm{~cm}$. Prior utilization, PDMS coupons were sonicated in a commercial detergent (Sonasol, Henkel Ibérica Portugal) for about 5 min, rinsed with distilled water for a few minutes, sonicated in methanol for about $20 \mathrm{~min}$, then rinsed with distilled water and air-dried overnight.

\subsection{Polydopamine Coating and Peptides Immobilization}

Coatings were prepared following two pDA-based approaches as illustrated in Figure 1, a two and a one-step immobilization. For

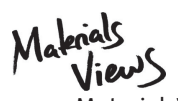

www.MaterialsViews.com 
A)

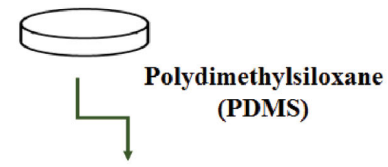

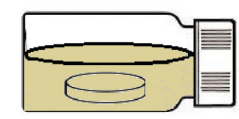

Dopamine + Polymyxin
Polymyxin immobilized (1-step PB/PE)

B)

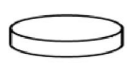

PDMS

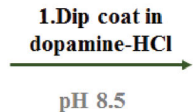

$\mathrm{pH} 8.5$

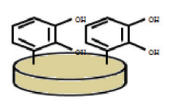

Polydopamine film (pDA)

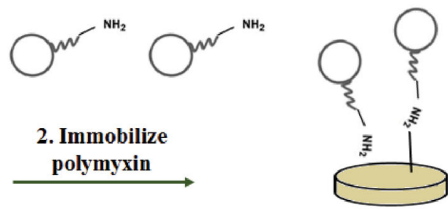

$\mathrm{pH} 8.5$

Polymyxin immobilized (2-step PB/PE)

Figure 1. Schematic representation of coating strategy developed for polymyxins immobilization onto PDMS material. A) PDMS was immersed in a solution containing dopamine and polymyxin together for one-step approach immobilization. B) For the two-step immobilization approach, PDMS was first functionalized with a layer of polydopamine, followed by polymyxins (PE or PB) immobilization.

peptides immobilization via the two-step approach (Figure 1B), the first step involved the deposition of a pDA coating on PDMS coupons which was performed by immersing them in $7 \mathrm{~mL}$ of a freshly prepared solution of dopamine (Sigma, $2 \mathrm{mg} \mathrm{mL}-1$ dopamine- $\mathrm{HCl}$ in $10 \times 10^{-3} \mathrm{M}$ bicine buffer, $\mathrm{pH} 8.5$ ) for $18 \mathrm{~h}$, at room temperature and under agitation (70 rpm). Coupons were then rinsed with ultrapure water. For further functionalization with peptides, pDA-coated coupons were immersed in PE or PB solutions ( 1 and $5 \mathrm{mg} \mathrm{mL}^{-1}$ in $10 \times 10^{-3} \mathrm{M}$ bicine buffer supplemented with $600 \times 10^{-3} \mathrm{M} \mathrm{NaCl}, \mathrm{pH}$ 8.5) and were incubated for $2 \mathrm{~h}$, at room temperature, under agitation (70 rpm). For the onestep polydopamine-based immobilization (Figure 1A), dopamine ( $2 \mathrm{mg} \mathrm{mL}^{-1}$ ) and polymyxins $\left(1 \mathrm{mg} \mathrm{mL}^{-1}\right)$ were dissolved together in $10 \times 10^{-3} \mathrm{M}$ bicine buffer solution ( $\mathrm{pH} 8.5$ ) and the PDMS coupons were immediately immersed in this solution. After overnight coating at room temperature and under agitation (70 rpm), the coupons were rinsed with ultrapure water and air-dried for $45 \mathrm{~min}$.

\subsection{Surface Characterization}

The surface morphology of materials was analyzed by scanning electron microscopy (SEM). Prior to observation, samples were sputter coated with gold and observed with an S-360 scanning electron microscope (Leo, Cambridge, MA, USA). SEM imaging was performed with the following parameters: $15 \mathrm{kV}$ accelerating voltage, $22 \mathrm{~mm}$ stage distance, 500x and 5000x magnification. Surface morphology and roughness were also evaluated using atomic force microscopy (AFM). AFM measurements were performed at room temperature using a Multimode with a Nanoscope III from Digital Instruments (USA) operating in tapping mode. Scan rates were set at $1 \mathrm{~Hz}$ and the scanning area per sample was fixed at $5 \mu \mathrm{m} \times 5 \mu \mathrm{m}$. Surface roughness analysis were conducted using NanoScope Analysis 1.10 software. The hydrophobicity parameters of material surfaces were determined using the sessile drop contact angle method, using an automated contact angle device (OCA 15 Plus, Dataphysics, Germany) that allows image acquisition and data analysis. Measurements were performed using $3 \mu \mathrm{L}$ drops of liquid on cleaned and dried coupons of PDMS before and after modifications. All measurements were performed at room temperature and water, glycerol and $\alpha$-bromonaphtalene were used as reference liquids for standardized contact angles measurements. Contact angles were related to the surface hydrophobicity, using the van Oss approach. ${ }^{[32]}$ According to van Oss, ${ }^{[33]}$ hydrophobicity can be expressed in the form of the free energy of interfacial interaction $\left(\Delta G_{\text {sws }}^{\text {TOT }}\right.$ ) between the particles of a solid surface (s), in an aqueous environment (w). According to this model, when $\Delta G_{\text {sws }}^{\text {TOT }}>0$, the surfaces are hydrophilic and for $\Delta G_{\mathrm{sws}}^{\mathrm{TOT}}<0$, they are hydrophobic.

\subsection{Peptides Immobilization Efficiency and Coatings Stability}

The efficiency of peptides immobilization was determined by quantifying the amount of unattached peptides in the buffer solution retrieved immediately after completing the coating process. The peptide concentration was measured using a fluorescamine (Sigma) assay. ${ }^{[34]}$ Briefly, before and after incubation of peptides onto PDMS (one-step immobilization approach) or pDA-coated PDMS coupons (two-step immobilization approach), the supernatants containing loaded and unattached peptides, respectively, were retrieved and used as samples to determine the peptide immobilization efficiency. Fluorescamine assay was performed by mixing fluorescamine solution $\left(3 \mathrm{mg} \mathrm{mL} \mathrm{m}^{-1}\right.$ in acetone) and the sample at 1:3 ratio in a 96-black-well plate (Greiner). After $15 \mathrm{~min}$ of incubation at room temperature, the fluorescence intensity of each sample was measured by using a microplate reader (Synergy HT, Biotek). Immobilization efficiency was represented as the percentage ratio of the amount of immobilized peptides to the amount of loaded peptides. Three independent assays with three replicates for each condition were performed. 
In order to investigate coatings stability, the detachment of immobilized peptides was quantified by measuring the amount of released peptides from the modified surfaces during incubation under a physiologically relevant condition (in PBS at $37^{\circ} \mathrm{C}$ ). For that, $500 \mu \mathrm{L}$ of a fresh PBS solution was added to each well of a 48-well microtiter plate (Orange Scientific, USA) in which PDMS coupons functionalized with peptides were placed immediately after peptide immobilization. The coupons were then incubated at $37{ }^{\circ} \mathrm{C}$ for $5 \mathrm{~d}$. Every day, supernatant samples $(500 \mu \mathrm{L})$ were withdrawn to determine the amount of peptide released. The remaining peptides on the surface were quantified by subtracting the released peptides from the total amount of peptides immobilized at the first day. These experiments were performed twice with three replicates for each condition tested.

\subsection{Bacterial Contact-Killing Assay}

In order to evaluate bacterial contact-killing properties of PDMS surfaces functionalized with polymyxins, a previously reported method was applied with some modifications. ${ }^{[35]}$ Briefly, bacterial concentration was adjusted in TSB to a final concentration of $1 \times 10^{6} \mathrm{CFU} \mathrm{mL} \mathrm{m}^{-1}$ and $20 \mu \mathrm{L}$ of this solution was added to each well of a 48-well microtiter plate, in which uncoated or modified PDMS coupons were placed. The plate was afterward incubated at $37{ }^{\circ} \mathrm{C}$, under static conditions for $24 \mathrm{~h}$. After that, materials were placed on a TSA plate, incubated for $24 \mathrm{~h}$ at $37^{\circ} \mathrm{C}$ and bacterial growth was checked for all conditions tested and tabulated as "+" for growth and "-" for no visible growth. Two independent assays with three replicates for each condition were performed.

\subsection{Bacterial Viability on Modified PDMS Surfaces}

Antibacterial performance of the generated surfaces against bacterial adhesion was evaluated by preparing a bacterial suspension with $1 \times 10^{8} \mathrm{CFU} \mathrm{mL} \mathrm{m}^{-1}$ in PBS from an overnight culture at $37{ }^{\circ} \mathrm{C}$. PDMS coupons were placed into the wells of a 48 -well tissue culture plate and covered with $300 \mu \mathrm{L}$ of bacterial suspension. The samples were kept at $37{ }^{\circ} \mathrm{C}$ and $120 \mathrm{rpm}$ for $4 \mathrm{~h}$, as this period of time has been reported to be enough for bacterial cells to achieve initial adhesion onto a surface. ${ }^{[36,37]}$ Samples were then washed with saline solution $(0.9 \% \mathrm{NaCl})$, stained with a live/dead stain (BacLight Bacterial Viability Kit, Invitrogen), and observed in a fluorescent inverted microscope (Leica, DMI 3000B). In this assay, the red-fluorescent nuclei acid staining agent propidium iodide, which only penetrates damaged cell membranes, was used to label dead bacterial cells on the PDMS surfaces. In contrast, the SYTO 9 green-fluorescent nucleic acid staining agent, which can penetrate cells both with intact and damaged membranes, was used to label viable cells. At least three images per coupon (a single image for each fluorescence channel) were collected and at least three coupons were inspected per assay. ImageJ (Version $1.49 \mathrm{~m}$, Wayne Rasband, National Institutes of Health, USA) software was used to subtract the image background and the threshold function was used to render each greyscale image into a binary translation with distinct areas identifying adhered bacteria. The threshold value supplied by ImageJ was used as default but when necessary the threshold value was manually adjusted until all visible cells were included within the thresholded range. The area measurement function was used to quantify the area of the pixels above the threshold and to thereby quantify the area covered by bacteria discriminating, at the same time, the fraction of live and dead bacteria, depending on the channel being analyzed. Values were normalized to PDMS control. Three independent assays with three replicates for each condition were performed.

\subsection{Susceptibility of Bacterial Cells Adhered to Modified Surfaces}

In order to evaluate the susceptibility pattern of bacterial cells adhered to PDMS surfaces, cells adhered on modified and unmodified surfaces were recovered and the MIC and MBC concentrations of peptide were afterward determined. Briefly, a bacterial suspension with $1 \times 10^{8} \mathrm{CFU} \mathrm{mL}^{-1}$ was prepared in PBS and $300 \mu \mathrm{L}$ of this suspension were added to a 48-well microtiter plate in which PDMS coupons were placed. The plate was incubated for $4 \mathrm{~h}$ at $37^{\circ} \mathrm{C}$ and $120 \mathrm{rpm}$. The coupons were subsequently washed three times with saline solution to remove free-floating bacteria and transferred to an eppendorf tube with $1 \mathrm{~mL}$ of saline solution. The tubes were then sonicated for $6 \mathrm{~min}$ and vortexed for $30 \mathrm{~s}$ for detaching the previous adhered cells and an aliquot of the bacterial suspension was added to MHB and used for MIC and MBC determination as aforementioned. Two independent assays with three replicates for each condition were performed.

\subsection{Cytotoxicity Assay}

Cytotoxicity tests were performed according to the ISO 109935:2006, using fibroblast cells 3T3 (CCL 163) obtained from ATCC. Cells were first cultured in Dulbecco's modified Eagle's medium (DMEM) supplemented with $10 \%$ of fetal bovine serum and $1 \%$ penicillin/streptomycin at $37{ }^{\circ} \mathrm{C}, 5 \% \mathrm{CO}_{2}$. After achieving the confluence, cells were detached using trypsin and $500 \mu \mathrm{L}$ of a cell suspension with $1 \times 10^{5}$ cells $\mathrm{mL}^{-1}$ were added to each well of a 48-well microtiter plate in which the PDMS materials were previously inserted. The plates were incubated at $37^{\circ} \mathrm{C}$ and $5 \% \mathrm{CO}_{2}$ for $48 \mathrm{~h}$. After that period of time, cytotoxicity was evaluated by the MTS (3-(4, 5-dimethylthiazol-2-yl)-5-(3-carboxymethoxyphenyl)2-(4-sulfophenyl)-2H-tetrazolium), inner salt reduction assay as a measure of cellular metabolic activity. All the medium was removed and a solution containing $100 \mu \mathrm{L}$ of MTS (Promega CellTiter 96 AQueous NonRadioactive Cell Proliferation Assay) per each $1 \mathrm{~mL}$ of DMEM without phenol red was added to each well. After $1 \mathrm{~h}$ of incubation in the dark, at $37{ }^{\circ} \mathrm{C}$ and $5 \% \mathrm{CO}_{2}$, the absorbance of the resulting solution was read at $490 \mathrm{~nm}$. Results were expressed as percentage of viable cells using the number of cells grown on pDA-coated PDMS as control. Two independent assays with three replicates for each condition were performed.

\subsection{Statistical Analysis}

Results were presented as mean \pm standard deviation (SD). Statistical analysis was performed by Kolmogorov-Smirnov normality test using Graph Pad Prism 5.0 for Mackintosh. After this analysis, parametric tests (one way Anova followed by Tukey's test) or nonparametric (Kruskal-Wallis test) were used depending on

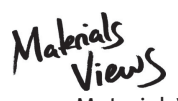

www.MaterialsViews.com 
whether the samples were from normally distributed populations or not, respectively.

\section{Results and Discussion}

With an ageing society, the problem of BAI is expected to increase in the coming years. When antimicrobial treatment fails, the removal of the infected implant may not completely solve the problem due to the remaining pathogen in the body, which is responsible for recurrent infections. ${ }^{[1,38]}$ Preventive approaches such as the modification of biomaterials to render them with antibacterial properties appear, therefore, as the best strategy to deal with these infections. In this study, pDA-mediated catechol functionalization was applied to render PDMS surfaces, a widely used biomaterial in clinical applications, with antimicrobial properties through the immobilization of two AMP: polymyxins B and E. Polymyxins $\mathrm{B}$ and $\mathrm{E}$ have been used as the last resort to fight multidrug resistant strains so there should be some caution in their widespread use to avoid the development of resistance which has already been reported. ${ }^{[24,39]}$ An alternative approach for their use that may minimize the potential development of microbial resistance as well as the toxicity toward mammalian cells relies on their covalent immobilization. ${ }^{[27]}$ Polymyxins B and E share many similarities regarding their mechanism of action, antimicrobial spectrum, clinical uses and toxicity. However, they also differ in several aspects, including chemical structure, formulation, potency, dosage, and pharmacokinetic properties. ${ }^{[40]}$ Their mechanism of action involves cell membrane's disruption by binding to the anionic part of the lipopolysaccharide of Gram-negative bacteria, which results in leakage of intracellular components. Because of its wider global availability, most clinical studies have been focused on PE. However, some studies have suggested that the incidence of nephrotoxic effects is higher with colistinmethate (the inactive form of $\mathrm{PE}$ ) than with $\mathrm{PB} \cdot{ }^{[41,42]}$

\subsection{MIC and MBC Determination on Planktonic Cultures}

The concentrations of polymyxins able to inhibit planktonic bacterial growth (MIC) and those required to kill planktonic bacteria $(\mathrm{MBC})$ were 2 and $4 \mu \mathrm{g} \mathrm{mL}^{-1}$, respectively, for both AMP.

\subsection{AMP Immobilization on PDMS Material}

In this study, AMP were immobilized onto PDMS and a pDA-based surface modification was applied for their immobilization using two different approaches (Figure 1). The two-step approach (Figure 1B), previously applied for the immobilization of other AMP, ${ }^{[43,44]}$ involved first the deposition of a uniform pDA coating from a dopamine$\mathrm{HCl}$ solution at a slightly alkaline $\mathrm{pH}$. The $\mathrm{pDA}$ coating was then used as a platform for AMP's immobilization due to the presence of residual quinones which present convenient sites for covalent grafting of nucleophilic groups such as amino functional groups found in AMP via Michael Addition and/or Shiff reactions. The thickness of these coatings, determined under similar conditions of this study using ellipsometry, was around $50 \mathrm{~nm} \cdot{ }^{[45,46]}$ Further functionalization with polymyxins should not alter this thickness.

For one-step pDA-based immobilization procedure (Figure 1A), the PDMS coupons were immersed in one-pot mixture of dopamine and the AMP to be immobilized. Previous work has shown that this procedure not only simplifies immobilization of biomolecules even further but it also increases the total amount of immobilized compounds at surfaces. ${ }^{[47]}$ To quantify the coating efficiency of peptides, the buffer solutions containing the unattached peptides were retrieved immediately after finishing the coating process. The percentage of peptide immobilized on PDMS (Table 1) was dependent on the approach used for polymyxins functionalization. Using a one-step approach, greater amounts of polymyxins B and E were immobilized (70\% and $61 \%$, respectively). However, taking into account that fluorescamine reacts with the primary amino groups

Table 1. Efficiency and stability of polydopamine-mediated immobilization of PE and PB. Stability was evaluated under physiologically relevant conditions (PBS at $37^{\circ} \mathrm{C}$ ) for $5 \mathrm{~d}$. ND means not determined.

\begin{tabular}{lcccccc}
\hline Method/polymyxin & \multicolumn{5}{c}{ Immobilized amount [\%] } \\
\cline { 2 - 6 } & Day 0 & Day 1 & Day 2 & Day 3 & Day 4 & Day 5 \\
\hline One-step [PE1] & $69.7 \pm 4$ & $69.2 \pm 0.4$ & $68.8 \pm 0.2$ & $68.4 \pm 0.1$ & $68.1 \pm 0.2$ & $68.1 \pm 0.1$ \\
Two-step [PE1] & $39.9 \pm 17$ & $39.0 \pm 0.5$ & $38.8 \pm 0.1$ & $37.8 \pm 0.8$ & $37.5 \pm 0.3$ & $37.3 \pm 0.3$ \\
Two-step [PE5] & $<0$ & & & ND & $60.1 \pm 0.2$ & $60.1 \pm 0.01$ \\
One-step [PB1] & $61.0 \pm 4$ & $60.8 \pm 0.1$ & $60.6 \pm 0.2$ & $60.4 \pm 0.1$ & $28.5 \pm 0.2$ & $28.5 \pm 0.1$ \\
Two-step [PB1] & $30.1 \pm 16$ & $30.0 \pm 0.1$ & $29.7 \pm 0.1$ & $28.8 \pm 0.3$ & ND &
\end{tabular}


found in the free amines on positively charged diaminobutyric acid residue of polymyxins, as well as the amine groups found on dopamine molecule in solution, it is hard to distinguish between the amount of dopamine polymerized and polymyxins immobilized using this onestep approach. Using the two-step approach, this limitation was overcome as dopamine polymerization occurred before polymyxins immobilization. For a lower concentration of loading polymyxins $\mathrm{B}$ and $\mathrm{E}\left(1 \mathrm{mg} \mathrm{mL}^{-1}\right)$ results showed a lower immobilization percentage of around $40 \%$ and $30 \%$, respectively. Increasing the loading concentration for $5 \mathrm{mg} \mathrm{mL}^{-1}$, the value of fluorescence measured after coating process was higher than the one obtained for the loading solution, yielding a percentage of immobilization lower than zero (Table 1). These results suggest that for a higher concentration, polymyxins may have interfered with the pDA coating, so that some amino groups present in this layer were removed from the PDMS surface, increasing, therefore, the content of amino groups detected by fluorescamine assay. Increasing peptide concentration increased the amount of amine groups that may have reacted with dopamine aggregates via noncovalent interactions. ${ }^{[48]}$ Therefore, it is reasonable to expect some changes in coating stability under aqueous conditions. However, in this study the PDMS functionalized with polymyxins using a loading concentration of $5 \mathrm{mg} \mathrm{mL}^{-1}$ retained its antimicrobial functionality, which is a sign that polymyxins were still grafted onto surfaces although some stability disturbance may have occurred.
In order to assess coatings stability, the detachment of immobilized polymyxins was quantified by measuring the amount of released polymyxin from the functionalized surfaces when incubated in PBS at $37{ }^{\circ} \mathrm{C}$. Results (Table 1) confirmed coatings stability using both strategies as the polymyxins did not significantly detach from the surfaces for up to $5 \mathrm{~d}$.

\subsection{Surface Characterization}

Surface morphology of pDA-mediated modified surfaces was characterized using SEM analysis (Figure 2). The unmodified PDMS exhibited smoother surface morphology compared with the modified ones. Self-polymerized PDA particles could be observed on modified PDMS coupons confirming the pDA coating. Further functionalization with polymyxins B or E yielded surfaces with different morphologies depending on the approach used. Results showed that one-step approach for immobilization of both polymyxins yielded surfaces with a more homogeneous coating with agglomerates more evenly distributed along the surfaces. This approach involves self-polymerization of dopamine in the presence of compounds to be immobilized, hence leading to homogeneous mixing of covalently linked compounds throughout the layer of $\mathrm{pDA}^{[30]}$ and surface characterization confirmed a more homogeneous coating. Moreover, while the amount of immobilized compounds via two-step approach is defined by the
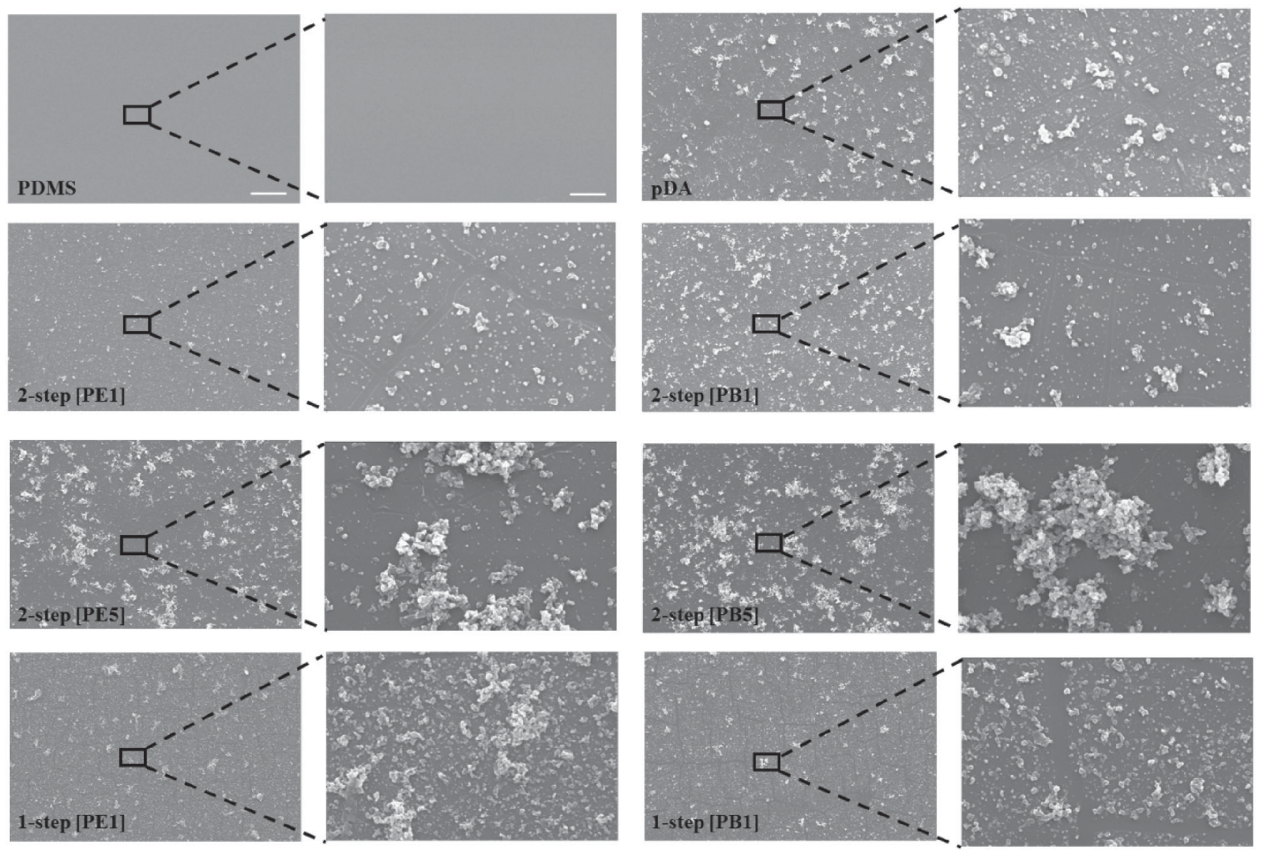

Figure 2. SEM images of unmodified polydimethylsiloxane surfaces (PDMS), polydopamine-coated PDMS (pDA), pDA-coated PDMS surfaces with immobilized PE and PB via two-step approach (two-step [PE] or two-step [PB]) and one-step approach (one-step [PE] and one-step $[\mathrm{PB}])$. The scale bars in the first and third column indicates $1 \mu \mathrm{m}$ and the bar scale in the second and fourth column indicates $10 \mu \mathrm{m}$.

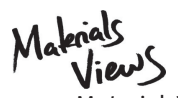

www.MaterialsViews.com 


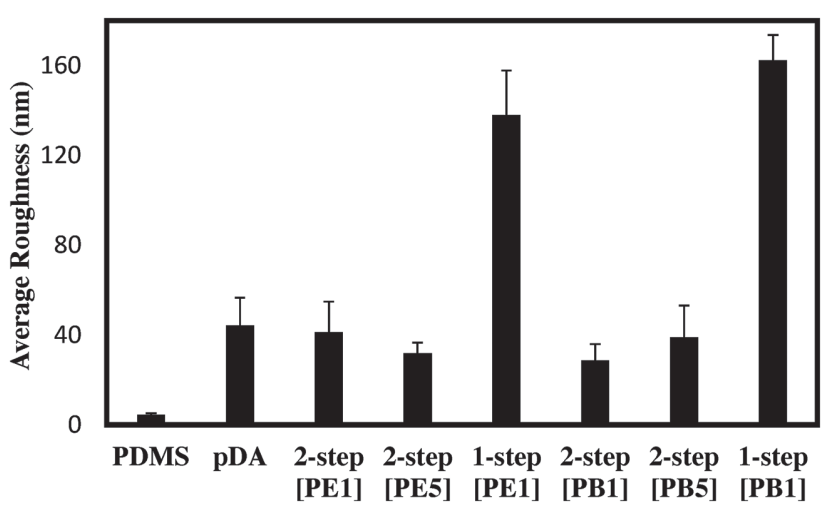

Figure 3. Average surface roughness $\left(R_{\mathrm{a}}\right)$ obtained from AFM analysis of unmodified polydimethylsiloxane surfaces (PDMS), polydopamine-coated PDMS (pDA), pDA-coated PDMS surfaces with immobilized PE and PB via two-step approach (two-step $[\mathrm{PE}]$ or two-step $[\mathrm{PB}]$ ) and one-step approach (one-step $[\mathrm{PE}]$ and one-step [PB]).

amount of reactive quinone groups that can react, which is limited to the surface of the outer surface, it is expected that biomolecules incorporation using onestep approach occurs throughout the full thickness of the pDA layer than only at its outer surface. ${ }^{[47]}$ When PE was immobilized using the two-step approach at a lower concentration, a similar morphology to the pDA coating alone was observed with smaller agglomerates. The increase of the loading concentration caused the formation of bigger agglomerates, heterogeneously distributed along the surface. For PB immobilization using the two-step approach, the same agglomeration formation could be observed.

For further surface morphology characterization, samples were also analyzed by AFM. From the AFM images, it was possible to measure the average roughness of
Table 3. Contact-killing activity of unmodified polydimethylsiloxane surfaces (PDMS), polydopamine-coated PDMS (pDA), pDAcoated PDMS surfaces with immobilized PE and PB via two-step approach (two-step [PE] or two-step [PB]) and one-step approach (one-step [PE] and one-step [PB]). Visible growth was used as an indicator of contact-killing activity and it was tabulated as "+" for growth and "-" for no visible growth.

\begin{tabular}{lc}
\hline Condition tested & Visible bacterial growth \\
\hline PDMS & + \\
pDA & + \\
Two-step [PE1] & - \\
Two-step [PE5] & - \\
One-step [PE1] & - \\
Two-step [PB1] & + \\
Two-step [PB5] & - \\
One-step [PB1] & +
\end{tabular}

surfaces (Figure 3). Results confirmed that the presence of a pDA layer increased the surface roughness, which is in agreement with other studies. ${ }^{[43,49]}$ Further functionalization with polymyxins E or B, using the two-step immobilization approach, did not interfere with surface roughness conferred by the pDA layer. On the other side, polymyxins immobilization using one-step approach yielded surfaces with significant higher values of roughness surface.

Hydrophobicity parameters of surfaces were investigated through contact angle measurements, using the van Oss approach. ${ }^{[33]}$ Contact angles, surface tension parameters, and free energy of interaction are presented in Table 2. Results show that PDMS exhibited a water contact angle higher than $65^{\circ}$ and a negative value of

Table 2. Values of contact angles $\left(^{\circ}\right)$ with water $\left(\theta_{\mathrm{W}}\right)$, glycerol $\left(\theta_{\mathrm{C}}\right), \alpha$-bromonaphtalene $\left(\theta_{\mathrm{B}}\right)$, surface tension parameters $\left(\mathrm{mJ} \mathrm{m}^{-2}\right)$, and free energy of interaction $\left(\Delta G_{\text {sws }}^{\text {TOT }}\right)\left(\mathrm{mJ} \mathrm{m}^{-2}\right)$ between the surfaces $(i)$ when immersed in water $(w)$. Values are means \pm SD.

\begin{tabular}{|c|c|c|c|c|c|c|c|}
\hline \multirow[t]{2}{*}{ Surface } & \multicolumn{3}{|c|}{ Contact angle $\left({ }^{\circ}\right)$} & \multicolumn{3}{|c|}{$\begin{array}{l}\text { Surface tension parameters } \\
\qquad\left(\mathrm{mJ} \mathrm{m}^{-2}\right)\end{array}$} & \multirow{2}{*}{$\begin{array}{c}\begin{array}{c}\text { Free energy of } \\
\text { interaction }\left(\mathrm{mJ} \mathrm{m}^{-2}\right)\end{array} \\
\left(\Delta G_{\mathrm{sws}}^{\mathrm{ToT}}\right)\end{array}$} \\
\hline & $\theta_{\mathrm{W}}$ & $\theta_{\mathrm{G}}$ & $\theta_{\mathrm{B}}$ & $\gamma_{\mathrm{i}}^{\mathrm{LW}}$ & $\gamma_{i}^{+}$ & $\gamma_{i}^{-}$ & \\
\hline PDMS & $108.6 \pm 3.2$ & $100.7 \pm 8.2$ & $55.9 \pm 6.0$ & 27.0 & 0 & 3.95 & -62.4 \\
\hline $\mathrm{pDA}$ & $56.6 \pm 4.8$ & $92.0 \pm 5.5$ & $13.6 \pm 5.7$ & 43.1 & 0 & 89.0 & 81.3 \\
\hline Two-step [PE1] & $77.4 \pm 10.4$ & $103.3 \pm 7.8$ & $28.8 \pm 8.0$ & 39.1 & 0 & 55.4 & 43.4 \\
\hline Two-step [PE5] & $84.6 \pm 11.6$ & $101.5 \pm 4.0$ & $28.5 \pm 7.1$ & 39.1 & 0 & 37.1 & 16.0 \\
\hline One-step [PE1] & $68.8 \pm 16.3$ & $117.3 \pm 6.3$ & $14.7 \pm 3.2$ & 43.0 & 0 & 104.5 & 97.4 \\
\hline Two-step [PB1] & $89.4 \pm 9.7$ & $100.4 \pm 3.3$ & $32.3 \pm 2.4$ & 37.8 & 0 & 26.7 & -1.9 \\
\hline Two-step [B5] & $87.8 \pm 9.3$ & $102.9 \pm 3.5$ & $28.1 \pm 4.4$ & 39.3 & 0 & 32.4 & 7.8 \\
\hline One-step [PB1] & $68.7 \pm 10.5$ & $117.0 \pm 9.7$ & $15.0 \pm 4.4$ & 42.9 & 0 & 104.2 & 97.1 \\
\hline
\end{tabular}


A)
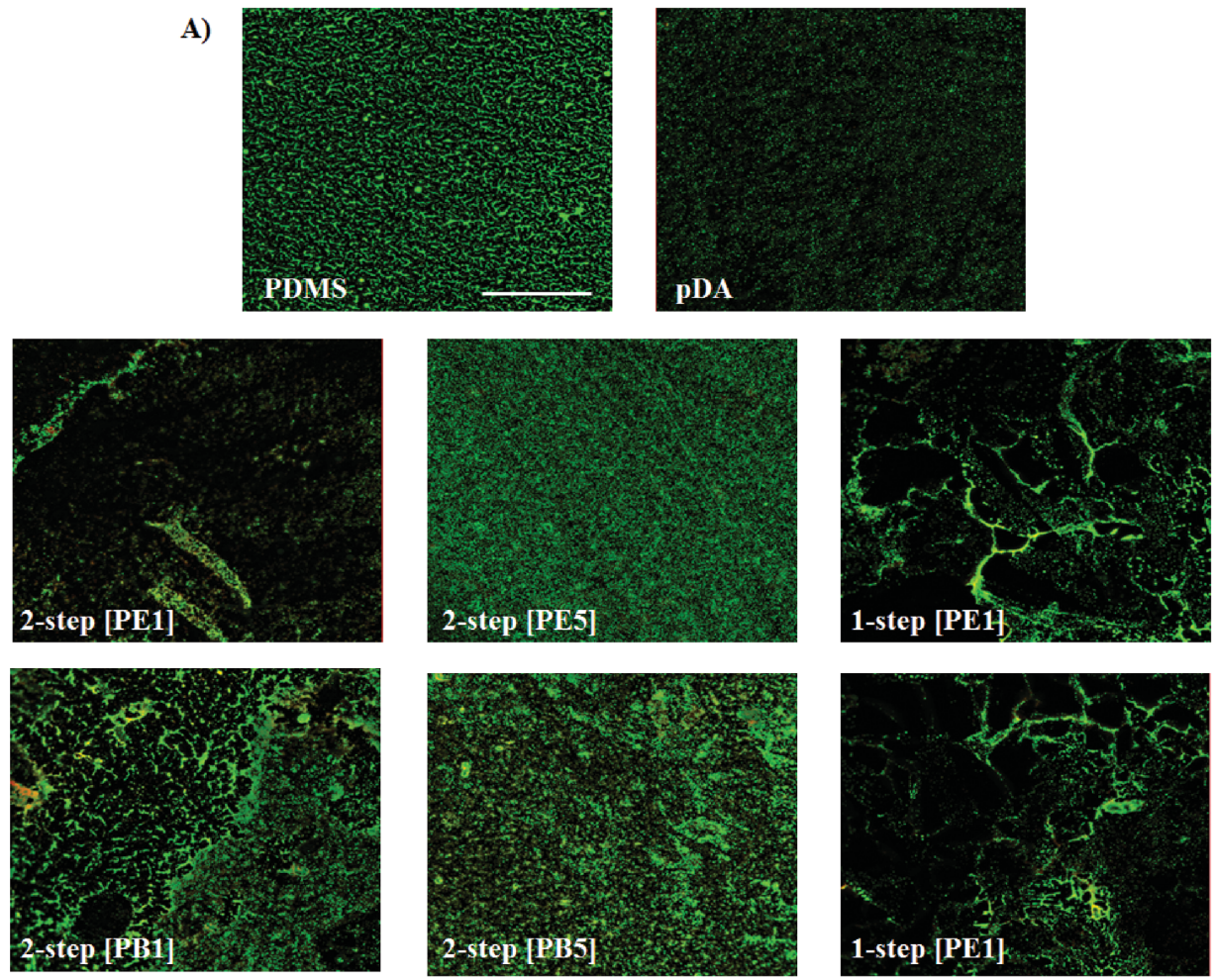

B)

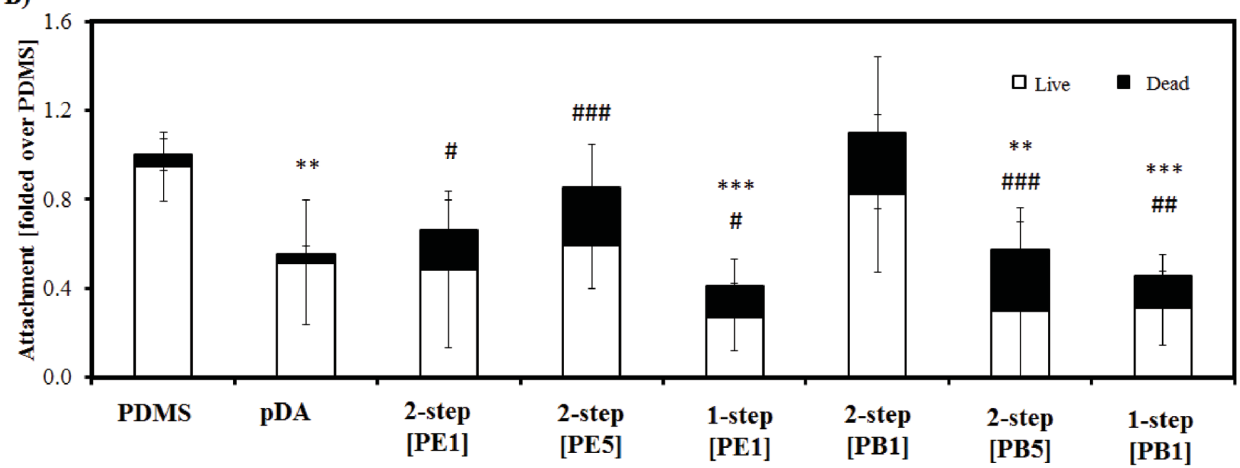

Figure 4. A) Representative fluorescent live/dead stain images obtained during P. aeruginosa ATCC 39324 attachment assays. The scale bar indicates $100 \mu \mathrm{m}$. B) Normalized attachment of $P$. aeruginosa ATCC 39324 onto unmodified polydimethylsiloxane surfaces (PDMS), polydopamine-coated PDMS (pDA), pDA-coated PDMS surfaces with immobilized PE and PB via two-step approach (two-step [PE] or twostep $[\mathrm{PB}]$ ) and one-step approach (one-step [PE] and one-step [PB]). All values were normalized to \% coverage on PDMS control. Significant differences were found for $\left({ }^{* *}\right) p<0.01$ and $\left({ }^{* * *}\right) p<0.001$, compared to PDMS control attachment and (\#\#) $p<0.01$ and (\#\#\#) $p<0.001$, compared to PDMS fraction of dead cells.

free energy of interaction $\left(\Delta G_{\text {sws }}^{\text {TOT }}\right)$, which are indicative of a hydrophobic feature. ${ }^{[50]}$ Further functionalization of PDMS yielded, in general, hydrophilic surfaces as indicated by the positive values of free energy of interaction $\left(\Delta G_{\mathrm{sws}}^{\mathrm{TOT}}\right)$. Polymyxin B immobilization using a two-step approach and a lower concentration (1 $\mathrm{mg}$ $\mathrm{mL}^{-1}$ ) was the only exception, presenting a hydrophobic character. Results also showed that although all the surfaces are electron donors, this feature was enhanced after pDA-based functionalization (higher values of $\left.\gamma_{i}^{+}\right)$.

\subsection{Antimicrobial and Anti-Adhesion Properties of PDMS Functionalized with Polymyxins}

Antibacterial performance of the generated surfaces was investigated against a mucoid reference strain of P. aeruginosa (ATCC 39324), as the production of alginate is one of the most extensively studied virulence factors. ${ }^{[51]}$ Contact-killing was evaluated by dropping a small volume of bacterial suspension on the surfaces of PDMS functionalized with polymyxins B or E for $24 \mathrm{~h}$ at $37^{\circ} \mathrm{C}$. Table 3 shows that no contact-killing was observed for bare PDMS

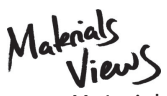

www.MaterialsViews.com 
and after pDA coating. Further functionalization with PB yielded surfaces with bacterial contact-killing activity but only when a higher concentration of this peptide (5 mg mL ${ }^{-1}$ ) was used during immobilization process. In turn, PDMS functionalized with PE exhibited bacterial contact-killing activity for both concentrations tested. When one-step immobilization approach was applied, only PE exhibited contact-killing activity.

For further evaluation of the antimicrobial performance of functionalized PDMS surfaces, an attachment assay was also performed in which bacteria were allowed to attach for $4 \mathrm{~h}$ and the remaining cells on the PDMS coupons were imaged with fluorescence microscopy. This period of time was chosen because the first $6 \mathrm{~h}$ after surgery (the so-called "decisive period") are identified as being critical for preventing bacterial adhesion in order to ensure the long-term success of the implant. ${ }^{[52]}$ During this period of time, there is a competition between integration of the material into the surrounding tissue and adhesion of bacteria to the implant surface. ${ }^{[53]}$ It was possible to measure the remaining cells on the modified surfaces and simultaneously discriminate between live and dead cells, or more, specifically, evaluate bacterial membrane's integrity (Figure 4). Unmodified PDMS material allowed the adhesion of $P$. aeruginosa cells and most of them remained alive. Polydopamine-coated surfaces slightly decreased the adhesion of this strain as compared to the unmodified PDMS but no significant antimicrobial effect was observed. Polymyxin E immobilization via two-step approach had no significant effect on bacterial attachment but was responsible for a higher fraction of dead cells. Increasing the concentration of PE during this two-step approach immobilization, had no effect on anti-adhesive or antimicrobial properties of the coating. On the other hand, when polymyxin E was immobilized during dopamine polymerization (one-step approach), bacterial attachment was decreased to the same levels as the ones achieved by pDA coating alone but a higher fraction of dead cells could be found. For PB immobilization via two-step approach, it was possible to conclude that the increase of the concentration enhanced the antimicrobial and anti-adhesive properties of the PDMS coupons. Polymyxin B immobilization via one-step approach yielded similar results to $\mathrm{PE}$ as it led to a reduction of bacterial attachment to the same level as the pDA coating alone and an increase of the fraction of dead cells.

\subsection{Susceptibility of Cells Adhered to the Modified Surfaces}

Although the resistance to polymyxins as well as to other AMP has been slower than to antibiotics, ${ }^{[54]}$ it has been showed that $P$. aeruginosa exposure to subinhibitory
Table 4. Susceptibility (MIC and MBC) of adhered cells to unmodified polydimethylsiloxane surfaces (PDMS), polydopamine-coated PDMS (pDA), pDA-coated PDMS surfaces with immobilized PE and PB via two-step approach (two-step [PE] or two-step [PB]) and one-step approach (one-step [PE] and one-step [PB]).

\begin{tabular}{lcc}
\hline $\begin{array}{l}\text { Cells recovered } \\
\text { from }\end{array}$ & MIC $\left(\mu \mathbf{~ m L}^{-1}\right)$ & MBC $\left(\mu \mathbf{~ m L}^{-\mathbf{1}}\right)$ \\
\hline PDMS & 2 & 8 \\
pDA & 2 & 8 \\
Two-step [PE1] & 2 & 8 \\
Two-step [PE5] & 1 & 2 \\
One-step [PE1] & 2 & 4 \\
Two-step [PB1] & 2 & 8 \\
Two-step [PB5] & 2 & 8 \\
One-step [PB1] & 2 & 8
\end{tabular}

levels of polymyxin B and $\mathrm{E}$ induces resistance toward higher, and otherwise lethal, levels of these antimicrobials. ${ }^{[39]}$ In order to evaluate if their covalent immobilization could overcome this issue, the potential development of bacterial resistance toward these modified surfaces was assessed. In this assay, cells in contact with unmodified PDMS and modified PDMS surfaces were recovered and used to determine the minimal inhibitory and bactericidal concentrations of polymyxins $B$ and E (Table 4). Results showed that cells adhered to PDMS functionalized with polymyxins B or E, using all the different approaches, exhibited the same or lower susceptibility pattern as cells adhered to PDMS or coated with $\mathrm{pDA}$, suggesting no development of resistance during this period of time. The higher $M B C$ found for adhered cells when compared to planktonic cultures was expected taking into consideration that adhered cells are inherently less susceptible than their planktonic counterparts. ${ }^{[55]}$ This first evidence is very important and promising, however, it should be taken into consideration that $4 \mathrm{~h}$ of adhesion may not be a sufficient period of time to conclude about resistance development. To strengthen the non-appearance of resistance, further studies should be performed where cells in contact with modified and unmodified surfaces should be continuously recovered and allowed to adhere to new samples during a longer period of time.

\subsection{Effect of PDMS Modified Surfaces on 3T3 Fibroblast Growth and Adhesion}

Another important concern associated to the use of polymyxins is their toxicity, ${ }^{[23]}$ so the knowledge of their effect on the human cells is also crucial. To predict the effects of the functional coatings developed in this study 


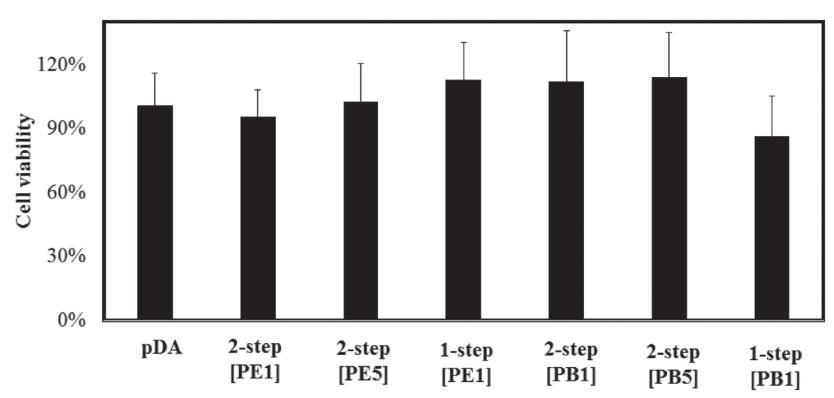

Figure 5. Viability of mammalian cells after $48 \mathrm{~h}$ of contact with unmodified polydimethylsiloxane surfaces (PDMS), polydopamine-coated PDMS (pDA), pDA-coated PDMS surfaces with immobilized PE and PB via two-step approach (two-step [PE] or two-step $[\mathrm{PB}]$ ) and one-step approach (one-step $[\mathrm{PE}]$ and onestep [PB]), measured with an MTS assay. Significant differences were not found for $p>0.5$ compared to polydopamine-coated PDMS surfaces (pDA).

on mammalian cells, a cytotoxicity assay was performed (Figure 5). Results showed that further functionalization of pDA-coated PDMS surfaces with both polymyxins had no significant effect on 3T3 fibroblast metabolic activity, which may be attributed to their covalent immobilization without leaching. Moreover, it should be emphasized that, although in the present study, a higher concentration of polymyxins ( 1 and $5 \mathrm{mg} \mathrm{mL}^{-1}$ ) was used for their immobilization, a much lower concentration was actually immobilized on the surfaces (about 40\%).

\section{Conclusions}

The emergence of multidrug resistant bacteria and the lack of alternative therapeutic options have led to the revival of polymyxins. Although effective, some concerns have been raised about its development of bacterial resistance and toxicity. The present work showed that their immobilization onto a surface greats hold potential to overcome these drawbacks. Immobilization of polymyxins B or E using a one-step pDA-based approach was able to prevent $P$. aeruginosa adhesion and kill a significant fraction of the adherent ones, without leaching from the surfaces and causing no harm to mammalian cells. Polymyxin E exhibited a better performance than $\mathrm{PB}$ as its immobilization onto PDMS generated surfaces with antimicrobial properties against $P$. aeruginosa, regardless the immobilization approach applied. The overall results showed that dopamine chemistry holds great potential for the immobilization of other AMP, without compromise their antimicrobial activity. In addition, the immobilization of PE holds great potential in the development of materials to fight BAI.

Acknowledgements: The authors acknowledge the Portuguese Foundation for Science and Technology (FCT), the strategic funding of UID/BIO/04469/2013 unit. This study was also supported by FCT and the European Community fund FEDER, through Program COMPETE, under the scope of the Projects "PTDC/SAU-SAP/113196/2009 (FCOMP-01-0124-FEDER-016012)," "RECI/BBB-EBI/0179/2012 (FCOMP-01-0124-FEDER-027462)," and "BioHealth-Biotechnology and Bioengineering approaches to improve health quality," Ref. NORTE-07-0124-FEDER-000027, co-funded by the Programa Operacional Regional do Norte (ON.2-O Novo Norte), OREN, FEDER. The authors also acknowledge FCT for the PhD Grant of Diana Alves (SFRH/BD/78063/2011).

Received: April 5, 2016; Revised: May 15, 2016; Published online: June 27, 2016; DOI: 10.1002/mabi.201600122

Keywords: antimicrobial peptides; antimicrobial surfaces; bacterial resistance; cytotoxicity; dopamine chemistry

[1] H. J. Busscher, H. C. van der Mei, G. Subbiahdoss, P. C. Jutte, J. J. van den Dungen, S. A. Zaat, M. J. Schultz, D. W. Grainger, Sci. Transl. Med. 2012, 4, 153rv110.

[2] D. Campoccia, L. Montanaro, C. R. Arciola, Biomaterials 2013, 34,8018 .

[3] L. Hall-Stoodley, J. W. Costerton, P. Stoodley, Nat. Rev. Microbiol. 2004, 2, 95.

[4] J. L. del Pozo, R. Patel, Clin. Pharmacol. Ther. 2007, 82, 204.

[5] H. C. Flemming, J. Wingender, Nat. Rev. Microbiol. 2010, 8, 623.

[6] S. de Bentzmann, P. Plesiat, Environ. Microbiol. 2011, 13, 1655.

[7] C. Ryder, M. Byrd, D. J. Wozniak, Curr. Opin. Microbiol. 2007, 10,644 .

[8] H. Nikaido, J. Bacteriol. 1996, 178, 5853.

[9] A. H. Tart, D. J. Wozniak, Curr. Top. Microbiol. Immunol. 2008, 322, 193.

[10] S. R. Shah, A. M. Tatara, R. N. D'Souza, A. G. Mikos, F. K. Kasper, Mater. Today 2013, 16, 177.

[11] S. He, P. Zhou, L. Wang, X. Xiong, Y. Zhang, Y. W. S. Deng, J. R. Soc., Interface 2014, 11, 1.

[12] L. A. T. W. Asri, M. Crismaru, S. Roest, Y. Chen, O. Ivashenko, P. Rudolf, J. C. Tiller, H. C. van der Mei, T. J. A. Loontjens, H. J. Busscher, Adv. Funct. Mater. 2014, 24, 346.

[13] P. Slepička, Z. Malá, S. Rimpelová, N. Slepičková Kasálková, V. Švorčík, React. Funct. Polym. 2015, 95, 71.

[14] P. Slepicka, N. S. Kasalkova, J. Siegel, Z. Kolska, L. Bacakova, V. Svorcik, Biotechnol. Adv. 2015, 33, 1120.

[15] D. Campoccia, L. Montanaro, C. R. Arciola, Biomaterials 2013, 34, 8533 .

[16] J. Hasan, R. J. Crawford, E. P. Ivanova, Trends Biotechnol. 2013, 31, 295.

[17] F. Costa, I. F. Carvalho, R. C. Montelaro, P. Gomes, M. C. Martins, Acta Biomater. 2011, 7, 1431.

[18] D. Alves, M. Pereira, Bioufouling 2014, 40, 483.

[19] M. Zasloff, Nature 2002, 415, 389.

[20] H. Altman, D. Steinberg, Y. Porat, A. Mor, D. Fridman, M. Friedman, G. Bachrach, J. Antimicrob. Chemother. 2006, 58, 198.

[21] M. Upton, P. Cotter, J. Tagg, Int. J. Microbiol. 2012, 2012, 1.

[22] M. E. Falagas, S. K. Kasiakou, Clin. Infect. Dis. 2005, 40, 1333.

[23] A. Michalopoulos, M. E. Falagas, Crit. Care Clin. 2008, 24, 377.

[24] M. E. Falagas, P. I. Rafailidis, D. K. Matthaiou, Drug Resist. Updat. 2010, 13, 132.

[25] N. C. Gordon, K. Png, D. W. Wareham, Antimicrob. Agents Chemother. 2010, 54, 5316.

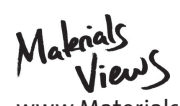

www.MaterialsViews.com 
[26] O. Cirioni, R. Ghiselli, C. Silvestri, W. Kamysz, F. Orlando, F. Mocchegiani, F. Di Matteo, A. Riva, J. Lukasiak, G. Scalise, V. Saba, A. Giacometti, Antimicrob. Agents Chemother. 2007, 51, 2005.

[27] D. Green, T. Fulghum, M. A. Nordhaus, in Science against Microbial Pathogens: Communicating Current Research and Technological Advances, Vol. 1 (Ed: A. Mendez-Vilas), Formatex Research Center, Badajoz, Spain, 2011, pp. 84-98.

[28] R. Bayston, L. E. Fisher, K. Weber, Biomaterials 2009, 30, 3167.

[29] L. Rodrigues, I. M. Banat, J. Teixeira, R. Oliveira, J. Biomed. Mater. Res. B Appl. Biomater. 2007, 81, 358.

[30] S. M. Kang, N. S. Hwang, J. Yeom, S. Y. Park, P. B. Messersmith, I. S. Choi, R. Langer, D. G. Anderson, H. Lee, Adv. Funct. Mater. 2012, 22, 2949.

[31] C. a. L. S. Institute, Methods for Dilution Antimicrobial Susceptibility Tests for Bacteria that Grow Aerobically, 6th ed., Vol. M7-A6, Wayne, Pennsylvania 2003.

[32] C. J. van Oss, Curr. Opin. Colloid Interface Sci. 1997, 2, 503.

[33] C. J. van Oss, R. F. Gies, Clays Clay Miner. 1995, 43, 474.

[34] E. Ko, K. Yang, J. Shin, S. W. Cho, Biomacromolecules 2013, 14, 3202.

[35] X. Ding, C. Yang, T. P. Lim, L. Y. Hsu, A. C. Engler, J. L. Hedrick, Y. Y. Yang, Biomaterials 2012, 33, 6593.

[36] D. J. Stickler, J. C. Lear, N. S. Morris, S. M. Macleod, A. Downer, D. H. Cadd, W. J. Feast, J. Appl. Microbiol. 2006, 100, 1028.

[37] R. Wang, K. G. Neoh, Z. Shi, E. T. Kang, P. A. Tambyah, E. Chiong, Biotechnol. Bioeng. 2012, 109, 336.

[38] A. F. Engelsman, I. C. Saldarriaga-Fernandez, M. R. Nejadnik, G. M. van Dam, K. P. Francis, R. J. Ploeg, H. J. Busscher, H. C. van der Mei, Biofouling 2010, 26, 761.

[39] Z. Yu, W. Qin, J. Lin, S. Fang, J. Qiu, Biomed. Res. Int. 2015, 2015, 1.

[40] A. Kwa, S. K. Kasiakou, V. H. Tam, M. E. Falagas, Expert Rev. Anti Infect. Ther. 2007, 5, 811.
[41] D. S. Akajagbor, S. L. Wilson, K. D. Shere-Wolfe, P. Dakum, M. E. Charurat, B. L. Gilliam, Clin. Infect. Dis. 2013, 57, 1300.

[42] R. L. Nation, J. Li, O. Cars, W. Couet, M. N. Dudley, K. S. Kaye, J. W. Mouton, D. L. Paterson, V. H. Tam, U. Theuretzbacher, B. T. Tsuji, J. D. Turnidge, Lancet Infect. Dis. 2015, 15, 225.

[43] K. Lim, R. R. Chua, H. Bow, P. A. Tambyah, K. Hadinoto, S. S. Leong, Acta Biomater. 2015, 15, 127.

[44] T. Shalev, A. Gopin, M. Bauer, R. W. Stark, S. Rahimipour, J. Mater. Chem. 2012, 22, 2026.

[45] H. Lee, S. M. Dellatore, W. M. Miller, P. B. Messersmith, Science 2007, 318, 426.

[46] P. Zhou, Y. Deng, B. Lyu, R. Zhang, H. Zhang, H. Ma, Y. Lyu, S. Wei, PLoS One 2014, 9, e113087.

[47] A. W. Nijhuis, J. J. van den Beucken, O. C. Boerman, J. A. Jansen, S. C. Leeuwenburgh, Tissue Eng. Part C Methods 2013, 19, 610.

[48] S. Hong, Y. Suk Na, S. Choi, I. Taek Song, W. Y. Kim, L. H,Adv. Funct. Mater. 2012, 22, 4711.

[49] D. R. Jun, S. K. Moon, S. W. Choi, Colloids Surf., B 2014, 121, 395.

[50] E. A. Vogler, Adv. Colloid Interface Sci. 1998, 74, 69.

[51] A. Boyd, A. M. Chakrabarty, J. Ind. Microbiol. 1995, 15, 162.

[52] K. A. Poelstra, N. A. Barekzi, A. M. Rediske, A. G. Felts, J. B. Slunt, D. W. Grainger, J. Biomed. Mater. Res. 2002, 60, 206.

[53] A. G. Gristina, Science 1987, 237, 1588.

[54] M. S. Paksu, S. Paksu, A. Karadag, G. Sensoy, N. Asilioglu, D. Yildizdas, B. N. Akyildiz, T. Kendirli, D. Demirkol, M. Akgun, E. Alp, E. Ciftci, A. K. Guney, N. Murat, Int. J. Antimicrob. Agents 2012, 40, 140.

[55] S. D. Aaron, W. Ferris, K. Ramotar, K. Vandemheen, F. Chan, R. Saginur, J. Clin. Microbiol. 2002, 40, 4172. 